\title{
Post Abortion Care and Management After Induced Abortion During the COVID-19 Pandemic: A Chinese Expert Consensus
}

Yu Wang · Qing Yang (D)

Received: November 12, 2020 / Accepted: December 14, 2020 / Published online: January 18, 2021

(C) The Author(s), under exclusive licence to Springer Healthcare Ltd. part of Springer Nature 2021

\section{ABSTRACT}

The rapid spread of novel coronavirus (COVID19) has posed complex challenges to global public health. During this pandemic period, access to essential services including post-abortion care (PAC) has been disrupted. Along with the clinical management of the disease in women, protection of the healthcare workers and medical staff from nosocomial infection is important to ensure infection control. Thus, in order to implement the proper contraceptive measures and to reduce the rate of repeated abortion, the family planning group of minimally invasive gynecological branch of the Liaoning Medical Association organized a committee of experts to formulate guidance and suggestions to ensure the timely treatment and surgery of women opting for abortion, the implementation of PAC, implementation of safe contraceptive measures after surgery, and the protection of healthcare professionals and medical staff from infection. We believe these guidelines might be helpful for obstetrics and gynecology departments in China and globally, as well for women who wish to undergo abortion during these unprecedented times.

Y. Wang · Q. Yang $(\bowtie)$

Department of Obstetrics and Gynecology,

Shengjing Hospital of China Medical University,

Shenyang, China

e-mail: yangqing_sj@126.com
Keywords: China; Contraception; Induced abortion; Infection; Novel coronavirus; Post abortion care

\section{Key Summary Points}

Along with the clinical management of the disease in women, protection of healthcare workers and medical staff from nosocomial infection is important to ensure infection control.

Healthcare workers should be trained to correctly wear and remove medical surgical masks and PPE, including gown or overall, face shields, and gloves.

Awareness regarding COVID-19 virus should be implemented by displaying related posters and publicity videos in the waiting area, and simultaneously educating the patients and their families on hand hygiene, respiratory hygiene, cough etiquette, disinfection and isolation, and disposal of medical waste.

Maximize the use of remote consultations (e.g., via video or telephone) to provide follow-up instructions related to postabortion care and assessment. 


\section{DIGITAL FEATURES}

This article is published with digital features, including a summary slide, to facilitate understanding of the article. To view digital features for this article go to https://doi.org/10.6084/ m9.figshare.13353317.

\section{INTRODUCTION}

The impact of novel coronavirus disease 2019 (COVID-19) has been increasing, with $14,562,550$ confirmed cases including 607,781 deaths globally, as reported by World Health Organization (WHO) on 21 July 2020 [1]. Further, in China, up to 21 July, according to reports from 31 provinces (autonomous regions and municipalities) and the Xinjiang Production and Construction Corps, there have been 233 confirmed cases (including 6 severe cases), a total of 78,840 cured and discharged cases, and a total of 4634 deaths [2]. The disease has been included as a class B infectious disease, as prescribed in the law of the People's Republic of China on the prevention and treatment of infectious diseases, and is managed as an infectious disease belonging to class A [3]. The pathogenic mechanism that produces pneumonia seems to be complex and associated with inflammatory response [4]. Viral infection is capable of producing an excessive immune reaction in the host, termed a 'cytokine storm', due to interleukin-6 [4].

Past global health emergencies have shown that access to safe abortion can be severely impacted due to delays in staff deployment and lack of infrastructure during crises [5]. Also, the effects of the pandemic can affect routine health care services, with patients waiting long hours at crowded clinics for antenatal care, contraceptive counseling, or other reproductive health services, thereby increasing the risk of infection transmission [5]. According to the WHO estimation, each year about 56 million induced abortions occur globally, of which nearly 7 million women in developing countries develop complications from unsafe abortions, and at least 22,000 women die from abortion-related complications [6]. The China
Health Statistics Yearbook 2018 reported that the overall trend of induced abortions for family planning in China showed a gradual growth from $30.0 \%$ in 1971 to $50.6 \%$ in 2017 [7]. Abortion is a time-sensitive service, and delays and denials will lead to unsafe abortions [8]. In ordinary times, access to abortion is essential because the choice may depend on a women's reproductive decision-making [9]. During the COVID-19 pandemic, such access becomes more important. Millions of women under quarantine may have limited access to contraception, many ambulatory clinics have restricted or limited visits, including those for placement of contraceptive devices, and women may have difficulty traveling safely to a pharmacy [10]. This pandemic has also resulted in substantial financial difficulties for many families, and not having the money to support a child is a leading reason that women choose to have an abortion [11]. Further, there is evidence that abortion rates are similar whether access to abortion is freely available or restricted; however, in cases of restricted access, women tend to resort to unsafe abortion outside medical regulation, which is likely to be detrimental to the health of the woman and require more specialist care from the healthcare system [12].

The current understanding of the clinical spectrum of the COVID-19 infection is limited. Transmission of the virus occurs mainly through respiratory droplets and close contact with the infected person $[3,13]$. However, less is known about the other potential transmission routes for COVID-19, like sexual and mother-toinfant transmission [14]. The WHO recommends counseling and the provision of contraceptive methods to all those women who wish to prevent unintended pregnancies and subsequent induced abortions [15]. In the time of the COVID-19 pandemic, WHO considers reproductive health services, including care during pregnancy and child birth, as essential services that need to continue [16]. To ensure safe and effective abortion care, greater use of remote consultations and medical abortion at home may be necessary, especially where women and staff may be self-isolating or acute hospital facilities are unavailable [17]. Therefore, guidelines for the safe management of women after 
post-abortion care (PAC) during the pandemic are needed along with proper training for the healthcare workforce. The National Population and Family Planning Commission Circular Printing and Distributing Action of China in 2018 issued the post-abortion contraceptive service specification, requiring hospitals at every level to carry out consultation, publicity, and implementation of contraceptive work during the peri-operative period of induced abortion [18]. Many hospitals have stopped PAC work due to the COVID-19 pandemic, which will inevitably affect the awareness regarding the need of contraception in women postabortion, and also affect the implementation rate of contraceptive methods. This will lead to repeated threat of abortion and ultimately affect reproductive health. Delay in providing abortion care will prevent women from being eligible for early medical abortion at home, will increase demand in operating theatres and at advanced gestation stage, and may result in more frequent complications, requiring a greater demand for specialist services that are already overstretched [17].

The family planning group of the minimally invasive gynecological branch of the Liaoning Medical Association organized a committee of experts to formulate guidance and suggestions to ensure the timely treatment and surgery of women opting for abortion, the implementation of PAC, implementation of safe contraceptive measures after surgery, and the protection of healthcare professionals and medical staff from infection. The consensus statements in this manuscript were framed based on the discussion by experts present at the meeting, considering both their personal experiences and the published literature for effective implementation of PAC in women who underwent induced abortion. Prior to the meeting, the planning group, consisting of experts including medical practitioners and medical publication experts in the relevant domains, was formed. To reach consensus, the organizing committee put forward problems that needed improvement in the PAC process based on the hospital management proposal during the COVID-19 pandemic issued by National Health Commission. The clinical experts then gathered to discuss and put forward a consensus based on the management experience of the hospital where the experts worked. The consensus process went through three rounds of discussions in which the experts listed different complications associated with induced abortions, their prevalence, the impact on services during the pandemic, and treatment regimens used for the management of PAC. Accordingly, recommendations were drafted in an effort to integrate infection control strategy and to promote professionalism in medical practice.

This article is based on previously conducted studies and personal experiences of the experts. It does not contain any new studies with human participants or animals performed by any of the authors.

\section{PREVENTIVE MEASURES FOR HEALTHCARE WORKERS FOR COVID-19 DISEASE}

Health workers are exposed to hazards that put them at high risk of infection at the outbreak of any disease. Hazards include pathogen exposure, long working hours, psychological distress, fatigue, occupational burnout, stigma, and physical and psychological violence [19]. Healthcare workers should handle the overall responsibility to ensure that all necessary preventive and protective measures are taken to minimize occupational safety and health risks [19]. China's National Health Commission has shown that more than 3300 health-care workers have been infected as of early March, of whom at least 22 personnel had died by end-February [20]. As the pandemic accelerated, access to personal protective equipment (PPE) for health workers and its shortage was a key concern [20]. Alongside concerns for their personal safety, healthcare workers have been anxious about passing the infection onto their families $[20,21]$.

WHO recommends that all healthcare workers, including frontline workers, should be trained for infection, prevention, and control (IPC) strategies to prevent or limit infection transmission in healthcare settings, including 
early recognition and source control, administrative controls, environmental and engineering controls, and PPE [22]. In general, standard precautions include hand and respiratory hygiene, the use of PPE according to a risk assessment, injection safety practices, safe waste management, proper clothes, environmental cleaning, and sterilization of patient-care equipment, and should be followed [23]. Universal precautions should be followed while dealing with all patients/their samples, irrespective of symptomatology [24]. Suspected COVID-19 patients should be contained in a separate area and should be given a medical mask while they are in waiting/public areas or in cohorting rooms [23]. Healthcare workers should follow the five-step practices for hand hygiene before and after examining any patient [25]. Further, proper use of PPE prevents contact with the spread of pathogens. It is important to ensure that environmental cleaning and disinfection procedures are followed consistently and correctly [26].

\section{Recommendations}

Healthcare personnel involved during this pandemic should be trained before reporting for duty. Healthcare staff should be trained in various aspects, such as the variety of modes of transmission and inactivation methods of the COVID-19 virus and medical protection, and continuously updated with relevant knowledge. Healthcare workers should be trained to correctly wear and remove medical surgical masks [27] and PPE, including gown or overall, face shields, and gloves [28, 29], to prevent contamination and to ensure occupational safety. All healthcare workers should be trained on hand hygiene and to follow the code of conduct on the work floor for the prevention of nosocomial infection when in direct and indirect contact with patient blood, body fluids, secretions (including respiratory secretions), and non-intact skin along with their surrounding environment. Hand hygiene should be included, which involves either cleaning hands with an alcoholbased hand wash or with soap and water. If there is no visible dirt on the hand, an alcohol- based hand wash is preferred for sanitizing the hands.

\section{ENSURING TRIAGE FOR EARLY INFECTION CONTROL OF DISEASE}

Clinical triage includes a system for assessing all patients at admission, which allows for early recognition of possible patients with suspected disease, and immediate isolation of those patients in an area separate from other patients [23]. To facilitate the early identification of cases of suspected COVID-19, there should be triage for outpatients. The triage should be implemented for all current and new patients presenting with critical illness, regardless of the diagnosis of COVID-19 or another illness [30]. Implementation of a triage system requires careful coordination between clinicians, health systems, local and regional governments, and the public, with a goal of transparency to maintain trust [30]. The absence of a triage system may lead to unnecessary deaths and increased moral distress for frontline healthcare workers, with more individuals being denied critical care, leading to overall worse outcomes across a population [30].

Guidelines recommend the presence of a well-equipped triage station at the entrance to the hospital facility, supported by trained staff [22]. Further, the institute should use screening questionnaires for the diagnosis of confirmed and suspected cases by referring to the Global Surveillance for human infection with coronavirus disease (COVID-19) for case definitions [22]. Also, signboards in public areas should be put up to remind symptomatic patients to alert healthcare workers [22]. The use of physical barriers, such as glass or plastic windows, can reduce exposure to the COVID-19 virus, [31]. This approach can be implemented in areas of the healthcare setting which patients will first enter, such as triage areas, the registration desk in the emergency department, or at the pharmacy window where medication is collected [31]. 


\section{Recommendations}

The establishment of triage before treatment is very important in order to avoid overcrowding of people outside the hospital or clinic during the pandemic. A three-level screening system should be implemented at every hospital and clinic for assessing safe abortion care for women. Screen and isolate all patients with suspected COVID-19 at the first point of contact with the healthcare system (such as the emergency department or outpatient department/clinic). In principle, suspected cases, probable cases, or confirmed cases should not be allowed to seek medical advice in the outpatient gynecology hospital or clinic. The firstlevel screening of the outpatient service should be set at the entrance of outpatient building facility. Patients entering the facility should wear masks and measure their body temperature upon arrival at the hospital. A facemask should be given to patients experiencing respiratory symptoms as soon as they get to the facility if they do not already have one. All patients in the "respiratory waiting area" should wear a facemask. If the body temperature of the patient is $\geq 37.3^{\circ} \mathrm{C}$, a healthcare staff shall be assigned for protection (secondary protection) [32], then the patient will be guided to the fever clinic for further investigation of the symptoms. Only patients whose body temperature is $<37.3^{\circ} \mathrm{C}$ will be allowed to enter the outpatient facility. Only one family member will be allowed to accompany each patient. The secondary screening should be set at the entrance of the waiting area of the hospital or clinic. Assign dedicated well-trained clinical staff for evaluation of patients presenting with respiratory symptoms. A standardized triage algorithm/questionnaire should be available for use, and should include questions that will determine if the patient meets the COVID-19 case definition. This should be filled in by the patient and their families to determine the clinical basis of the disease. Patients whose body temperature is $<37.3^{\circ} \mathrm{C}$ and with no history of epidemiological contact will be allowed in the waiting area for treatment. If the body temperature is detected to be $>37.3^{\circ} \mathrm{C}$, or those who have a history of epidemiological contact, will be led to the fever clinic by a specially assigned person for further clinical investigation. The third-level screening can be set up in the consulting room, and only patients who clear the first and second levels of screening will be allowed to consult the gynecologist. In addition to epidemiological investigation and measurement of body temperature, a pre-examination triage table should be set up especially for the pandemic period. One doctor or provider should treat 1 patient at a time in the consulting room, and the consulting room should be disinfected after use, twice a day. For those women who do not seek termination of pregnancy and do not suffer from complications, it is recommended that patients seek medical treatment as close as possible to their area of residence to avoid gathering at "large hospitals" and avoid exposure to infection. If there is no necessity for emergency surgery, the surgery of intrauterine device (IUD) insertion and removal should be delayed according to the patients' condition. For those who are suspected of inevitable abortion, incomplete abortion, missed abortion, or other complications, timely treatment should be ensured. Patients and the accompanying family member are required to wear masks throughout the whole process. It is advisable for the patient to not change the accompanying family members. When the accompanying family member is changed, reexamination for epidemiological history and body temperature will be carried out. Figure 1 describes in detail the outpatient triage process.

\section{MANAGEMENT OF OUTPATIENT AREA AND PATIENT AWARENESS REGARDING COVID-19}

Studies have reported that environmental surfaces are more likely to be contaminated with the COVID-19 virus in healthcare settings where certain medical procedures are performed [33-35]. Environmental controls should be maintained to reduce the spread of pathogens and contamination on surface and inanimate objects. The measures include spatial separation of at least $1 \mathrm{~m}$ between each patient and others, including healthcare workers [22, 23, 31]. WHO 


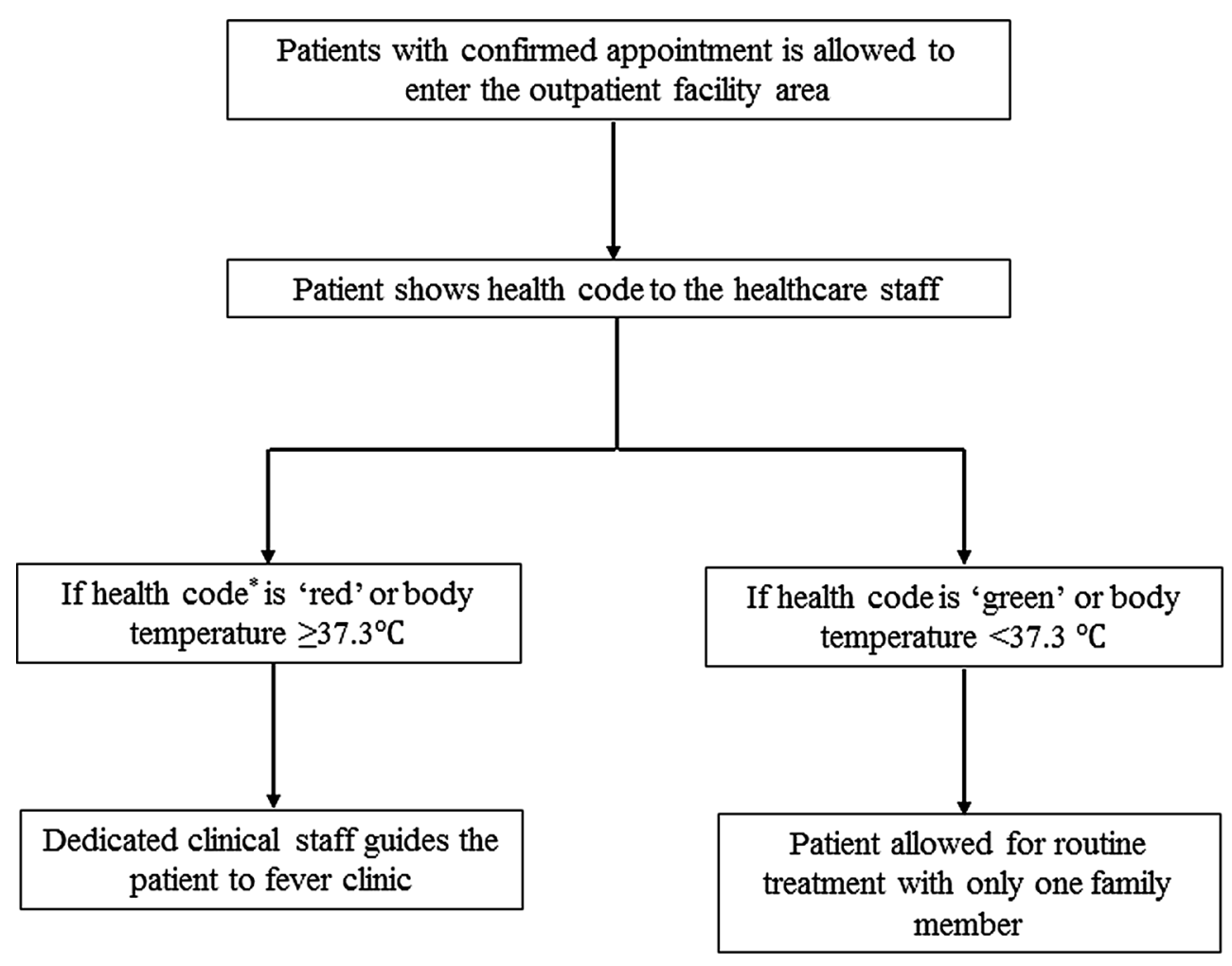

*Health code records a patients' joumaey: give users colour-coded designations based on their health status and travel history.

A green code users are allowed to travel relatively freely. A yellow code indicates that the user should be in home isolation, and a red code indicates the useris a confirmed Covid-19 patient and should be in quarantine.

Fig. 1 Outpatient triage process

recommends various frequencies of cleaning of environmental surfaces, according to the patient areas with suspected or confirmed COVID-19 patients [36]. Thoroughly cleaning environmental surfaces with water and detergent and applying commonly used hospitallevel disinfectants (such as sodium hypochlorite) are effective and sufficient procedures [36]. Medical devices and equipment, laundry, food service utensils, and medical waste should be managed in accordance with safe routine procedures $[37,38]$. Administrative controls should be followed which include developing sustainable IPC infrastructures and activities, educating patient caregivers, developing policies on the early recognition of acute respiratory infection potentially caused by COVID-19 virus, facilitated access to laboratory testing, prevention of overcrowding especially in emergency departments, providing dedicated waiting areas for symptomatic patients and isolating hospitalized patients, provision of adequate training for healthcare workers, and maintaining adequate patient-to-staff ratios [22, 31, 38].

\section{Recommendations}

Patients and only one family member will be allowed to wait in the outpatient waiting area. The number of patients and their family members in the waiting area should be reduced to avoid over-gathering. When the number of people in the waiting area exceeds the defined number specified in the protocol, the nurses in the waiting area will alert the registration help desk and the entry checkpoint to control the entry of patients and their family members. A spatial separation $(>1 \mathrm{~m}$ ) should be maintained between the patients and their families and between the public and hospital staff [22]. Further, the hospital staff should educate the patients and their families to dispose of surgical 
masks and other medical wastes after use into yellow-colored garbage cans. Medical institutions should carry out air disinfection or be equipped with circulating air disinfection equipment [39]. When there are visible pollutants to the naked eye, the disposable absorbent material will be used to remove the pollutants and then the disinfection of the entire floor should be carried out. The cleaned pollutants can be disposed in a centralized way according to the provisions mentioned in the "Medical Waste Management Regulations" [39]. The floor should be disinfected with $500 \mathrm{mg} / \mathrm{L}$ chlorinebased disinfectant twice a day. During the surgery, a notice board with the caption "the floor is wet and slippery, be careful do not fall" should be placed to prevent any healthcare personnel or patients from falling over on slippery surfaces. Awareness regarding COVID-19 virus can be implemented by displaying related posters and publicity videos in the waiting area, and simultaneously patients and their families should be educated regarding this virus, including precautionary measures regarding hand hygiene, respiratory hygiene, cough etiquette, disinfection and isolation, and disposal of medical waste [40]. Patients as well as healthcare staff and other personnel should be trained on the use of surgical masks, PPE, including gloves, face shields, or protective masks, and proper use of disinfectants [40].

While providing education to patients and their families on PAC during COVID-19 disease, an open waiting area with a limited number of people should be maintained. The PAC education should emphasize the importance of longterm contraception and its effectiveness to avoid unintended pregnancies. Also, emphasis should be given on how to adhere to short-term contraception for maximum benefit and reduce the risk of unintended pregnancy caused by contraceptive failure. Any awareness campaigns/classes in the closed environment should be avoided, and alternative ways can be resorted to, such as the use of mobile applications like WeChat, social media, and other forms of communications. For PAC clinics that do not engage in collective education, outpatient consultation and individual consultation is recommended so that the rate of implementation of contraceptive measures after surgery will not be affected.

\section{POST-ABORTION CARE TREATMENT AND INDEPENDENT CONSULTATION}

For women requiring abortion services, WHO recommends alternate modes of abortion services, including self-management of medical abortion up to 12 weeks' gestation, to avoid exposure to infection [41]. Medical management of induced abortion at $<12$ weeks of gestation is recommended with the use of $200 \mathrm{mg}$ mifepristone administered orally, followed 1-2 days later by $800 \mu \mathrm{g}$ misoprostol or the use of $800 \mu \mathrm{g}$ misoprostol administered vaginally, sublingually, or buccally. For women undergoing abortion at $\geq 12$ weeks of gestation, $200 \mathrm{mg}$ mifepristone administered orally, followed 1-2 days later by repeat doses of $400 \mu \mathrm{g}$ misoprostol or repeat doses of $400 \mu \mathrm{g}$ misoprostol administered vaginally, sublingually, or buccally every $3 \mathrm{~h}$ is recommended by WHO [42].

NICE guidelines recommend that women access accurate information by consulting the healthcare provider via remote consultations, i.e., via video or telephone $[17,43]$. The gynecologist should discuss the various options for contraceptive use with the patient. Further, various guidelines recommend that the healthcare provider includes a method of contraception in the treatment pack (e.g., an oral contraceptive), especially when a women's ability to use long-acting reversible contraception will be impacted due to travel and other restrictions [17, 44]. Only women requesting an early medical abortion and contraception will be required to attend in person in cases where the benefit of doing so outweighs the risk of COVID-19 exposure and transmission [17, 44]. In China, women are prohibited from self-induced abortion with medications which must only be carried out in qualified medical institutions. This step is necessary as the intake of medication results in massive bleeding especially during the first $10-12$ weeks postabortion. 


\section{Recommendations}

The consulting gynecologist should ensure firstlevel protection (including protective mask, hand gloves, and surgical cap) when examining the patient. In cases where the patient is suspected of COVID-19 disease, a second level of protection should be ensured (PPE, face shield or goggles, surgical mask) [32]. A temporary warning belt should be placed to separate the distance between the gynecologist and patients $(>1 \mathrm{~m})$. During the visit, the "one doctor, one patient, one consulting room" protocol should be strictly followed, and the activity of the staff in the consultation area should be strengthened. Due to the implementation of the new set of rules, there may be delays in the waiting period which might lead to inconvenience among the patients and their families present in the waiting area. Efficient personnel must be stationed in the consulting area to pacify the patients and their families for the expected delay, and contact the emergency response group in case any unreasonable disturbance arises.

Due to the rise of pandemic situation, the gynecologist should discuss the option of postoperative contraception methods along with routine consultation for surgery. The gynecologist should explain the reasons for the failure of contraception, clarify the need for contraception, provide appropriate post-operative contraceptive options to women opting for induced abortion, and strengthen the necessity for safe contraceptive options for the safe health of the patient. For outpatients who are suspected of not following-up routinely or of following the process of contraception, the gynecologist should strive for the timely implementation of contraception after surgery. Long-term contraceptive measures should be implemented simultaneously during the surgery for those who do not wish to give birth for the next 2 years after the termination of pregnancy. For those women who cannot implement long-term contraceptive measures, it is recommended that they follow at least 3 months of highly-effective contraceptive measures (such as the combined oral contraceptive pill), and ensure that the patient continues the medications for another 3 months in cases of no obvious side effects and complications. In order to reduce the number of visits of patients to the hospital and to improve the implementation rate of long-term contraceptive methods, medical abortion is not recommended for those who do not suffer from high-risk factors during the pandemic. Also, prior to the surgery, the surgeon should provide complete, accurate, and easy-to-understand information about the procedure, and what to expect during and afterwards, to woman in a format that is accessible to her and helps with her decision-making and voluntary consent. Information regarding post-abortion contraception also needs to be provided [45]. PAC can be organized via video or teleconferencing consultations and other networks in cases where the patient does not develop any complications.

In the past, independent (one-to-one) consultation was an important and key link in the implementation of effective and long-term contraceptive measures. However, during the pandemic, the gynecologist should focus on providing treatment on a case-by-case basis. Relevant case report forms, including details such as pregnancy and childbirth history, surgery history, etc., should be prepared and handed to women visiting the hospital or clinic. Based on the details filled in by every woman, the consulting gynecologist can make a decision. This will shorten the time of individual consultation, avoid crowd gathering, help identify the key reasons of contraceptive failure according to the differential service of consultation between patients and outpatient doctors, and implement contraceptive measures according to the individual condition of the patients. For patients who have understood and agree to follow contraceptive measures at the time of treatment, independent consultation can be delayed. 


\section{PATHWAYS TO MANAGEMENT OF FOLLOW-UP AFTER INDUCED ABORTION}

Under normal conditions, a routine follow-up visit is recommended by WHO only in cases where misoprostol alone is used for the complete abortion procedure. Routine follow-up is not necessary after an uncomplicated surgical or medical abortion using mifepristone and misoprostol; however, women may be offered an optional follow-up visit 7-14 days after their procedure to provide further contraceptive counseling or to address any medical concerns [46]. At the follow-up appointment, a patient's recovery process will be evaluated along with any signs or symptoms of ongoing pregnancy, reviewing any available medical records, performing a focused physical examination in response to any complaints, and assessing the patient's fertility goals and need for contraceptive services. If no method was started prior to discharge from the facility, further information and counseling for appropriate contraceptive methods will be provided by the gynecologist $[42$, 46]. In China, it is suggested that all patients, including those opting for induced abortion, should be routinely followed-up at 2 weeks, 1 month, 3 months, 6 months, and 12 months post-operation [18].

\section{Recommendations}

According to the PAC follow-up recommendations issued by the National Health Commission, the follow-up period of induced abortion under normal conditions is divided into shortterm follow-up and long-term follow-up [18]. The short-term follow-up is considered as 2 weeks and 1 month after the surgery, while the long-term follow-up period can be 3, 6, and 12 months after the surgery. The short-term follow-up will help to understand the postpartum recovery in women and the process of menstruation, evaluate whether there is residual intrauterine adhesions and contraceptive use, answer patients' questions, and provide supplementary contraceptives. The long-term follow-up after surgery should be carried out to understand the use and compliance of contraceptive methods, whether there was a second unexpected pregnancy, and to give guidance and promotion for further consultation. However, during the pandemic, routine visits to the hospital or clinic are not allowed and patients can access alternative follow-up services. For example, maximize the use of remote consultations (e.g., via video or telephone) to provide follow-up instructions related to PAC and assessment. If the patient does not take the initiative to consult on the follow-up date, the hospital staff should contact the patient to inquire about post-operative bleeding, contraceptive method use, etc., and provide follow-up guidance on contraceptive methods. It is recommended that women who have opted for IUD insertion during the time of surgical abortion should follow-up at their nearest gynecological center or hospital after menstruation to avoid unwanted pregnancy due to the displacement of the IUD within the uterine cavity. Figure 2 represents the comparison of the changes of PAC workflow during the pandemic and during normal conditions.

Patients with suspected post-operative complications should be informed that they should return to hospital as soon as possible. For patients with residual diagnosis, an individualized treatment regimen should be introduced depending on the patient situation. Conservative treatment is recommended for women who meet the following criteria: (1) intrauterine residual tissue diameter $<2.5 \mathrm{~cm}$; (2) residual time of tissue $<21$ days; (3) blood human chorionic gonadotropin (HCG) $<400 \mathrm{mIU} / \mathrm{ml}$; and (4) no obvious signs of infection. The conservative treatment for induced abortion includes the use of mifepristone with or without the combination of misoprostol for 1-2 weeks post-surgery and after observation of clear residue discharge on ultrasound scanning. In cases of massive vaginal bleeding or acute abdomen and other clinical manifestations, the patient is advised to go to the nearest emergency department for consultation. During the standard treatment, it is recommended to clear the uterus if there is a large amount of vaginal bleeding, or there is a tissue in the uterine cavity, or there is residue after menstruation, to avoid the risk of 


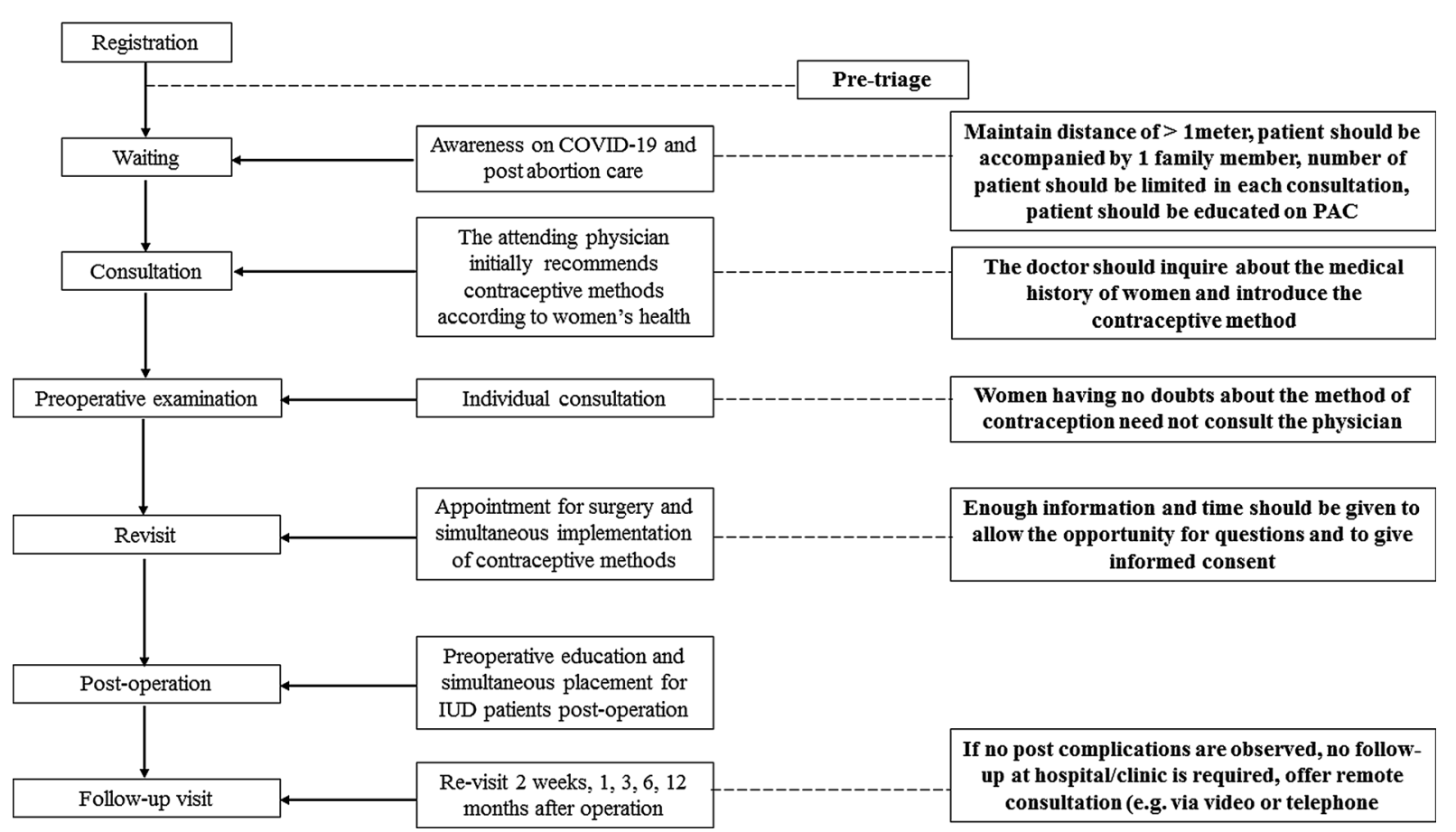

Fig. 2 Comparison of PAC workflow between normal and pandemic period

infection. For women who experience delayed menstruation but confirm intended pregnancy post-surgery, a cycle of progesterone and estrogen can be initiated to avoid miscarriage. If there is no menstruation after one cycle of treatment, hysteroscopy should be carried out to eliminate intrauterine adhesions. After induced abortion, many patients may develop endometritis, which can further develop into myometritis, appendicitis, pelvic peritonitis, and even septicemia $[47,48]$. Therefore, women suspected of having a post-operative infection are recommended to return to hospital for timely treatment.

\section{KEY CONSENSUS RECOMMENDATIONS}

- Abortion is time-sensitive and attention should be paid to provide care as early as possible.

- Healthcare workers should be trained to correctly wear and remove medical surgical masks [27] and PPE, including gown or overall, face shields, and gloves [28, 29].
- Awareness regarding COVID-19 virus should be implemented by displaying related posters and publicity videos in the waiting area and simultaneously educating the patients and their families on hand hygiene, respiratory hygiene, cough etiquette, disinfection and isolation, and disposal of medical waste [40].

- Post-abortion treatment can be decided by the healthcare provider on a case-by-case basis, and is recommended to delay unless the patient develops any post-surgery complications requiring immediate attention.

- Maximize the use of remote consultations (e.g., via video or telephone) to provide follow-up instructions related to post-abortion care and assessment.

\section{CONCLUSION}

Management of essential services including post-abortion care during the pandemic COVID-19 disease in outpatients is crucial. Further, it is equally important to ensure the safety of the healthcare workers and medical 
staff from nosocomial infection to ensure infection control. The PAC work due to the COVID-19 pandemic should not be delayed to prevent the repeated threat of abortion in women of childbearing age. Implementation of PAC during difficult times will integrate the strength of the whole hospital, carry out all prevention and control work in an orderly, effective, quick and safe manner according to the work requirements, and ensure a normal surgery of induced abortion and the timely implementation of contraceptive measures.

\section{ACKNOWLEDGEMENTS}

The authors would like to thank all the experts of family planning group of Minimally Invasive Gynecology branch of Liaoning Medical Association.

Funding. No funding or sponsorship was received for this study or publication of this article. The Rapid Service Fee was funded by the authors.

\section{Medical Writing and Editorial Assis-} tance. Further, the author would like to acknowledge Anwesha Mandal, Dr. Kaushik Subramanian and Dr Amit Bhat, Indegene Pvt Ltd., India for their medical writing support. Medical writing assistance was funded by the authors.

Authorship. All named authors meet the International Committee of Medical Journal Editors (ICMJE) criteria for authorship for this article, take responsibility for the integrity of the work as a whole, and have given their approval for this version to be published.

Disclosures. Yu Wang and Qing Yang have nothing to disclose.

Compliance with Ethics Standards. This article is based on previously conducted studies and personal experiences of the experts. It does not contain any new studies with human participants or animals performed by any of the authors.
Data Availability. Data sharing is not applicable to this article as no datasets were generated or analyzed during the current study.

\section{REFERENCES}

1. WHO Coronavirus Disease (COVID-19) Dashboard [Internet]. [cited 2020 Jul 22]. Available from: https://covid19.who.int

2. The latest situation of the novel coronavirus pneumonia epidemic as of $24: 00$ on July 21 [Internet]. [cited $2020 \mathrm{Jul}$ 22]. Available from: http:// www.nhc.gov.cn/xcs/yqtb/202007/ 13f951072dfa4c3a8ebf396e308847d7.shtml

3. Pei-Fang W. Diagnosis and Treatment Protocol for Novel Coronavirus Pneumonia (Trial Version 7). Chin Med J. 2020;133:1087-95.

4. Cascella M, Rajnik M, Cuomo A, Dulebohn SC, Di Napoli R. Features, Evaluation and Treatment Coronavirus (COVID-19). StatPearls [Internet]. Treasure Island (FL): StatPearls Publishing; 2020 [cited $2020 \mathrm{Jul}$ 17]. Available from: http://www. ncbi.nlm.nih.gov/books/NBK554776/

5. Hussein J. COVID-19: What implications for sexual and reproductive health and rights globally? Sex Reproduct Health Mater. 2020;28:1746065.

6. KeyAbortionFactsFinal.pdf [Internet]. [cited 2020 Jul 23]. Available from: http://www.redaas.org.ar/ archivos-recursos/KeyAbortionFactsFinal.pdf

7. Liu J, Englert Y, Zhang W-H. Is Induced Abortion a Part of Family Planning in China? Induced Abortion and Spontaneous Early Pregnancy Loss - Focus on Management [Internet]. 2019 [cited 2020 Jul 23]; Available from: https://www.intechopen.com/ books/induced-abortion-and-spontaneous-earlypregnancy-loss-focus-on-management/is-inducedabortion-a-part-of-family-planning-in-china

8. Todd-Gher J, Shah PK. Abortion in the context of COVID-19: a human rights imperative. Sex Reproduct Health Mater. 2020;28:1758394.

9. Chae S, Desai S, Crowell M, Sedgh G. Reasons why women have induced abortions: a synthesis of findings from 14 countries. Contraception. 2017;96:233-41.

10. Bayefsky MJ, Bartz D, Watson KL. Abortion during the Covid-19 pandemic-ensuring access to an essential health service. N Engl J Med. 2020;382: e47. 
11. Finer LB, Frohwirth LF, Dauphinee LA, Singh S, Moore AM. Reasons U.S. women have abortions: quantitative and qualitative perspectives. Perspect Sex Reproduct Health. 2005;37:110-8.

12. Sedgh G, Bearak J, Singh S, Bankole A, Popinchalk A, Ganatra B, et al. Abortion incidence between 1990 and 2014: global, regional, and subregional levels and trends. Lancet. 2016;388:258-67.

13. General Office of National Health Committee. Office of State Administration of Traditional Chinese Medicine. Notice on the issuance of a program for the diagnosis and treatment of novel coronavirus (2019-nCoV) infected pneumonia (trial sixth edition). [Internet]. [cited $2020 \mathrm{Jul} \mathrm{1].} \mathrm{Available}$ from: http://yzs.satcm.gov.cn/zhengcewenjian/ 2020-02-19/13221.html

14. The COVID-19 Outbreak: Potential Fallout for Sexual and Reproductive Health and Rights [Internet]. Guttmacher Institute. 2020 [cited 2020 Jul 23]. Available from: https://www.guttmacher.org/ article/2020/03/covid-19-outbreak-potential-

fallout-sexual-and-reproductive-health-and-rights

15. Alkema L, Chou D, Hogan D, Zhang S, Moller A-B, Gemmill A, et al. Global, regional, and national levels and trends in maternal mortality between 1990 and 2015, with scenario-based projections to 2030: a systematic analysis by the UN Maternal Mortality Estimation Inter-Agency Group. Lancet. 2016;387:462-74.

16. Organization WH. COVID-19: operational guidance for maintaining essential health services during an outbreak: interim guidance, 25 March 2020. 2020 [cited $2020 \mathrm{Jul} 24$ ]; Available from: https://apps. who.int/iris/handle/10665/331561

17. Coronavirus (COVID-19) infection and abortion care, Information for healthcare professionals [Internet]. [cited 2020 Aug 2]. Available from: https:// www.rcog.org.uk/globalassets/documents/ guidelines/2020-07-31-coronavirus-covid-19infection-and-abortion-care.pdf

18. (Notice on printing and distributing contraceptive service specification after induced abortion (2018 Edition), August 13, 2020 [Internet]. [cited 2020 Sep 3]. Available from: http://www.nhc.gov.cn/fys/ s7904/201808/

c211b0059b1447119bdf4f0a5128de50.shtml

19. Coronavirus disease (covid-19) outbreak: rights, roles and responsibilities of health workers, including key considerations for occupational safety and health [Internet]. [cited 2020 Aug 3]. Available from: https://www.who.int/docs/defaultsource/coronaviruse/who-rights-roles-respon-hwcovid-19.pdf?sfvrsn=bcabd401_0
20. Lancet T. COVID-19: protecting health-care workers. The Lancet. 2020;395:922.

21. Mental health and psychosocial considerations during the COVID-19 outbreak [Internet]. [cited 2020 Aug 3]. Available from: https://www.who.int/ docs/default-source/coronaviruse/mental-healthconsiderations.pdf?sfvrsn=6d3578af_2

22. Infection prevention and control during health care when novel coronavirus ( $\mathrm{nCoV}$ ) infection is suspected [Internet]. [cited 2020 Aug 2]. Available from: https://www.who.int/publications-detailredirect/10665-331495

23. Organization WH. Infection prevention and control during health care when COVID-19 is suspected: interim guidance, 19 March 2020. 2020 [cited 2020 Aug 2]; Available from: https://apps. who.int/iris/handle/10665/331495

24. EssentialservicesduringCOVID19updated0411201. pdf [Internet]. [cited 2020 Aug 2]. Available from: https://www.mohfw.gov.in/pdf/

EssentialservicesduringCOVID19updated0411201. pdf

25. World Health Organization, editor. WHO guidelines on hand hygiene in health care: first global patient safety challenge: clean care is safer care. Geneva, Switzerland: World Health Organization, Patient Safety; 2009.

26. environmental-cleaning-RLS-H.pdf [Internet]. [cited 2020 Aug 2]. Available from: https://www. cdc.gov/hai/pdfs/resource-limited/environmentalcleaning-RLS-H.pdf

27. Zuo S, Chen Y, Zeng C, An-hua WU. Introduction to the application scope and related standards of masks in various countries. Chin J Infect Control 19, 109-16.

28. Li Liu-yi Wu, An-Hua. Confusion on prevention and control of healthcare-associated infection of novel coronavirus. Chin J Infect Control. 2020;19: $105-8$.

29. Ye Li, Cai Ran Lu, Ye. Selection and use of new protective clothing against coronavirus pneumonia. Chin J Infect Control. 2020;19:117-22.

30. Maves RC, Downar J, Dichter JR, Hick JL, Devereaux A, Geiling JA, et al. Triage of scarce critical care resources in COVID-19 an implementation guide for regional allocation. Chest. 2020;158:212-25.

31. Rational use of personal protective equipment for coronavirus disease 2019 (COVID-19) [Internet]. [cited 2020 Aug 2]. Available from: https://apps. who.int/iris/bitstream/handle/10665/331215/ WHO-2019-nCov-IPCPPE_use-2020.1-eng.pdf 
32. Lang Jinghe, Zhu Lin. Chinese expert consensus: prevention and control of gynecological diagnosis and treatment during the new corona virus epidemic [Internet]. [cited 2020 Aug 2]. Available from: http://www.cogonline.com/info/ 132278742790816989

33. Faridi S, Niazi S, Sadeghi K, Naddafi K, Yavarian J, Shamsipour M, et al. A field indoor air measurement of SARS-CoV-2 in the patient rooms of the largest hospital in Iran. Sci Total Environ. 2020;725: 138401.

34. Ye G, Lin H, Chen S, Wang S, Zeng Z, Wang W, et al. Environmental contamination of SARS-CoV-2 in healthcare premises. J Infect. 2020;81:e1-5.

35. Ong SWX, Tan YK, Chia PY, Lee TH, Ng OT, Wong MSY, et al. Air, Surface Environmental, and Personal Protective Equipment Contamination by Severe Acute Respiratory Syndrome Coronavirus 2 (SARS-CoV-2) From a Symptomatic Patient. JAMA. 2020;323:1610-2.

36. Cleaning and disinfection of environmental surfaces in the context of COVID-19 [Internet]. [cited 2020 Aug 4]. Available from: https://www.who.int/ publications-detail-redirect/cleaning-anddisinfection-of-environmental-surfaces-inthecontext-of-covid-19

37. WHO | Decontamination and Reprocessing of Medical Devices for Health-care Facilities [Internet]. WHO. [cited 2020 Aug 2]. Available from: http:// www.who.int/infection-prevention/publications/ decontamination/en/

38. World Health Organization, Pandemic and Epidemic Diseases, World Health Organization. Infection prevention and control of epidemic- and pandemic-prone acute respiratory infections in health care: WHO guidelines. [Internet]. 2014 [cited 2020 Aug 2]. Available from: http://apps.who.int/ iris/bitstream/10665/112656/1/9789241507134_ eng.pdf?ua $=1$

39. Technical Guidelines for Prevention and Control of Novel Coronavirus Infection in Medical Institutions (First Edition) [Internet]. [cited 2020 Aug 3]. Available from: http://www.nhc.gov.cn/yzygj/ s7659/202001/

b91fdab7c304431eb082d67847d27e14.shtml
40. Guidelines for the Use of Common Medical Protective Products in the Prevention and Control of Pneumonia from New Coronavirus Infection (Trial) [Internet]. [cited 2020 Aug 3]. Available from: http://www.nhc.gov.cn/yzygj/s7659/202001/ e71c5de925a64eafbe1ce790debab5c6.shtml

41. Clinical management of COVID-19 [Internet]. [cited 2020 Aug 4]. Available from: https://www. who.int/publications-detail-redirect/clinicalmanagement-of-covid-19

42. World Health Organization, Reproductive Health and Research. Medical management of abortion [Internet]. 2018 [cited 2020 Aug 4]. Available from: http://www.ncbi.nlm.nih.gov/books/NBK536779/

43. NICE. Abortion Care. National Institute for Health and Care Excellence (NICE). NG140. 25-9-2019. London, NICE. NICE Guideline. [Internet]. [cited 2020 Aug 4]. Available from: https://www.nice.org. uk/guidance/ng140

44. PAPAC Statement - Covid 19 and Postabortion Care.pdf [Internet]. [cited 2020 Aug 4]. Available from: https://reproductiverights.org/sites/default/ files/2020-05/PAPAC\%20Statement $\% 20-\%$ 20Covid\%2019\%20and\%20Postabortion\%20Care. pdf

45. Safe abortion: technical and policy guidance for health systems. Reproductive Health Matters. 2012;20:205-7.

46. Information NC for B, Pike USNL of M $8600 \mathrm{R}, \mathrm{MD}$ B, Usa 20894. Clinical Practice Handbook for Safe Abortion. Post Abortion. [Internet]. World Health Organization; 2014 [cited 2020 Aug 4]. Available from: https://www.ncbi.nlm.nih.gov/books/ NBK190099/

47. Sajadi-Ernazarova KR, Martinez CL. Abortion Complications. StatPearls [Internet]. Treasure Island (FL): StatPearls; 2020 [cited 2020 Aug 3]. Available from: http://www.ncbi.nlm.nih.gov/ books/NBK430793/

48. Rouse CE, Eckert LO, Muñoz FM, Stringer JSA, Kochhar S, Bartlett L, et al. Postpartum endometritis and infection following incomplete or complete abortion: case definition and guidelines for data collection, analysis, and presentation of maternal immunization safety data. Vaccine. 2019;37: 7585-95. 